\title{
Efficient BIST Hardware Insertion with Low Test Application Time for Synthesized Data Paths
}

\author{
Nicola Nicolici and Bashir M. Al-Hashimi \\ School of Engineering and Advanced Technology, Staffordshire University \\ Stafford ST18 0AD, U.K. \\ Email: nicola@bss10.staffs.ac.uk
}

\begin{abstract}
In this paper, new and efficient BIST methodology and BIST hardware insertion algorithms are presented for RTL data paths obtained from high level synthesis. The methodology is based on concurrent testing of modules with identical physical information by sharing the test pattern generators in a partial intrusion BIST environment. Furthermore, to reduce the number of signature analysis registers and test application time the same type modules are grouped in test compatibility classes and n-input k-bit comparators are used to check the results. The test application time is computed using an incremental test scheduling approach. An existing test scheduling algorithm is modified to obtain an efficient trade-off between the algorithm complexity and testable design space exploration. A cost function based on both test application time and area overhead is defined and a tabu search-based heuristic capable of exploring the solution space in a very rapid time is presented. To reduce the computational time testable design space exploration is carried out in two phases: test application time reduction phase and BIST area reduction phase. Experimental results are included confirming the efficiency of the proposed methodology.
\end{abstract}

\section{Introduction}

Built-in self-test BIST is a design-for-testability (DFT) technique in which testing is accomplished through builtin hardware [1]. Pseudo-random BIST techniques use linear feedback shift registers (LFSRs) to perform test pattern generation (PG), whilst multiple-input signature registers (MISRs) carry out signature analysis (SA). Builtin logic block observation (BILBO) registers [2] are used to perform either PG or SA, and concurrent BILBOs (CBILBOs) perform PG and SA simultaneously. Today's need for a shorter time to market makes manual insertion of BIST circuitry an unaffordable design bottleneck and synthesis-based approaches were adopted.

An early approach to add BIST structures to register transfer-level (RTL) circuit is presented in [3]. This approach does not take into account the test application time. A latter approach that minimizes test application time and area overhead is presented in [4]. The number of test plans examined for each module was limited to four which leads to inefficient testable design space exploration. A different approach based on simultaneous test hardware insertion and test scheduling at RTL is presented in [5]. While previous test scheduling approaches $[6,7,8]$ assume fixed test resource allocation which implies that the test hardware is allocated before the test scheduling process, this work presents an incremental test scheduling procedure where test scheduling is performed concurrently with test hardware allocation. A branch and bound technique is employed during the exploring process to prune the design space. This means that complex designs like a 32 point discrete cosine transform (DCT), with a high number of feasible testable solutions will be explored in a very long time. Therefore, more sophisticated methods like iterative improvement techniques are required to explore the testable design space more efficiently. A different approach $[9,10]$ based on testability metrics like randomness and transparency [11] considers test path generation and test scheduling. This strategy lacked of a global view of the testable design space and the suboptimal final solution depends on the order in which the modules are processed. Recently Stroele et al. [12] proposed simultaneous test hardware insertion and test scheduling at the gate-level. This approach also minimizes test control and test application time. Highly efficient algorithms are presented for the gate-level description, which has a much higher 
complexity than RTL descriptions. The suboptimal test hardware insertion caused by the low complexity of RTL descriptions can be overcome by the fact that same type modules share the same physical information which is the focus of this paper. Furthermore the sharing of test pattern generators and reuse of signature analysis registers, as presented in our paper, can also solve the problems of minimal test application time, low BIST area overhead and efficient test schedule at RTL.

\section{Preliminary definitions}

A RTL data path consists of modules (functional resources), registers and multiplexers. Let $n_{\text {reg }}, n_{\bmod }$ and $n_{\text {res }}$ denote the number of registers, modules and moduletypes. Portions of data path, such as multiplexers and registers, can easily be tested using functional patterns. The goal of partial intrusion BIST is to test all the modules using a subset of registers in the test mode. Any register that is connected to an input port of a module through only multiplexers can supply test patterns to that input port. The embeddings where both input ports of a module receive test patterns from the same test register are not valid in the proposed BIST methodology. The correlation between test patterns at the left and right input port would decrease the number of unique test patterns that could be applied, hence decreasing the fault coverage. Any register that collects data from the output port of a module through only multiplexers is a possible choice for modification as a BIST register to compress test responses. Because modules of the same type share the identical physical information a decrease in the test application time can be obtained by grouping modules of the same type into test compatibility classes (TCCs). A test compatibility class $T C C_{i, j}$ of a module type $j$ is the set of modules that can be tested at the same time under the current test register configuration. If a test register generates test patterns for the left input port of the functional module $M_{i}$ and for the right input port of the functional module $M_{j}$, it is considered a shared test resource and modules $M_{i}$ and $M_{j}$ cannot belong to the same test compatibility class. Similarly different-type modules cannot belong to the same test compatibility class due to the fact that their fault sets have different detection probability profiles. Each module-type $j$ has $n_{\text {classes }}(j)$. All the modules from a $T C C_{i, j}$ receive the same test patterns. The output responses of all the modules from $T C C_{i, j}$ are compared by an n-input k-bit comparator, where $n$ is $\left|T C C_{i, j}\right|$ and $k$ is the bit-width of the data path. If two output signals differ, an error signal is activated to the BIST controller. If output responses of all the modules are the same, a single register compresses the responses in order to verify if this succession of output responses is the correct one. Let $O R S\left(M_{k}\right)$ denote the set of output registers of module $M_{k}$. The output register set of a test compatibility class $\operatorname{ORS}\left(T C C_{i, j}\right)$ is the union of output register sets of all the modules from $T C C_{i, j}$. The register that performs the signature analysis function for $T C C_{i, j}$ is chosen from $\operatorname{ORS}\left(T C C_{i, j}\right)$. Thus, instead of performing compression of test responses for $n_{\text {mod }}$ modules, the test response compression is performed for only $n_{\text {res }} x \sum n_{\text {classes }}(j)$ test compatibility classes. The $n$-input $k$-bit comparator logic can be tested by a short deterministic test set, in the same way as multiplexers. It should be noted when $n_{\text {mod }}=n_{\text {res }}$ the proposed BIST methodology is identical with the BIST embedding methodology $[3,4,5]$ where each module is embedded between two test pattern generators and a signature analysis register.

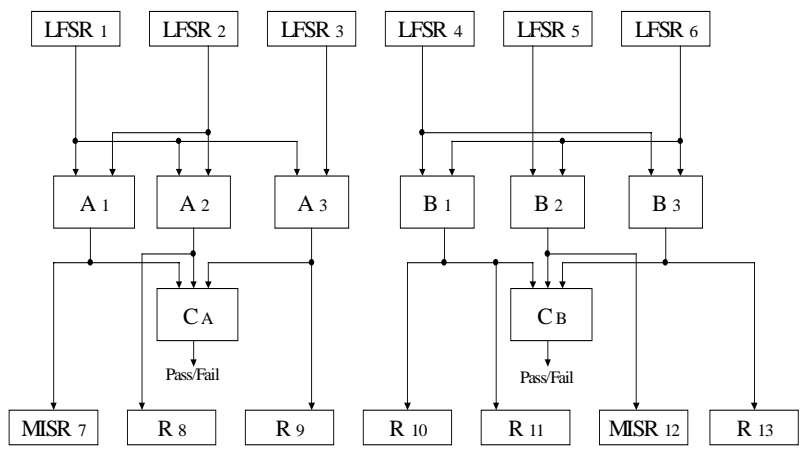

\section{Figure 1. Data path example to illustrate the proposed BIST methodology}

To illustrate the efficiency of RTL data path testing using TCCs consider the data path example of Figure 1, where $L F S R_{1}, L F S R_{2}$ and $L F S R_{3}$ test three modules of $A_{\text {type }}, L_{F S R_{4}}, L F S R_{5}$ and $L F S R_{6}$ test three modules of $B_{\text {type }}$. $L F S R_{I}$ generates test patterns for the left input port of $A_{l}$, $A_{2}$ and $A_{3}$. $L F S R_{2}$ generates test patterns for the right input port of $A_{1}$ and $A_{2}$, whilst $L F S R_{3}$ provides test patterns for the right input port of $A_{3}$. Modules $A_{1}, A_{2}$ and $A_{3}$ belong to $T C C_{0,0}$ (class index 0 and $A_{\text {type }}$ index is 0 ). Similarly, modules $B_{1}, B_{2}$ and $B_{3}$ belong to $T C C_{0,1}$ (class number 0 and $B_{\text {type }}$ index is 1). Given the bit-width of the data path as 8 bits, the output responses of the modules from $T C C_{0,0}$ are compared by a 3 -input 8 -bit comparator $\left(C_{A}\right)$. Also, the output responses of modules from $T C C_{0,1}$ are compared by another 3 -input 8 -bit comparator $\left(C_{B}\right)$. If any error occurs during testing, the Pass/Fail signal will be activated and the testing process will stop. The $O R S\left(A_{1}\right)$ is $\left\{R_{7}\right\}$, the $\operatorname{ORS}\left(A_{2}\right)$ is $\left\{R_{8}\right\}$, and the $\operatorname{ORS}\left(A_{3}\right)$ is $\left\{R_{9}\right\}$. Hence the $\operatorname{ORS}\left(T C C_{0,0}\right)$ is $\left\{R_{7}, R_{8}, R_{9}\right\}$. Any of these registers can be configured as signature analysis register for $T C C_{0,0}$. The procedure that chooses the optimal signature analysis register is presented in section 4.2. For 
data path example in Figure 1 the chosen register is $R_{7}$. Similarly, the $\operatorname{ORS}\left(T C C_{0,1}\right)$ is $\left\{R_{10}, R_{11}, R_{12}, R_{13}\right\}$ and the chosen signature analysis register is $R_{l 2}$.

\section{Local neighbourhood search of the testable design space}

Having defined the BIST methodology based on test compatibility classes the testable design space must be explored using an efficient BIST hardware insertion algorithm. The BIST hardware insertion algorithm looks for a testable solution with a primary goal of minimizing test application time and as a second order goal to minimize BIST hardware overhead. The testable design space exploration phases are detailed in section 5. This section focuses on the local neighbourhood of the testable design space and on generation of new neighbour solutions. In our problem formulation the current solution is a partially testable design where only the test pattern generators are allocated. The fully testable design is generated for each solution using the algorithms from section 4 . The testable design space of partially testable designs is explored using a tabu search-based heuristic. Tabu search was proposed as a general combinatorial optimization technique [13]. This heuristic is similar to iterative improvement in that moves are sought which transform the current solution to its best neighbouring solution. The algorithm starts with an initial solution obtained by randomly assigning a single test pattern generator to every input port of every module from the RTL data path. Tabu search maintains a tabu list of its $r$ most recent moves, with $r$ circuit-dependent and varying from 5 (small designs) to 10 (complex designs). The tabu list exists to prevent cycling near a local minimum and also to enable uphill moves. In our problem formulation for a RTL data path with $n_{\text {reg }}$ registers there are $n_{\text {reg }}$ neighbour solutions (one for each register from the data path). Each register $R_{i}$ can generate test patterns to input port $I P$ of $n_{i}$ modules of module-type $F R_{j}$. The neighbour solution for register $R_{i}$ is obtained by moving the test pattern generation-function for input port $I P$ of the $n_{i}$ modules of module-type $F R_{j}$ modules to $R_{i}$ and removing the test pattern generator-function from registers that have previously generated test patterns for the respective $n_{i}$ modules. When generating a neighbour solution for each register $R_{i}$ there are a maximum of $2 \times n_{\text {res }}$ potential moves (one for each input port of every module-type for which the respective register can generate test patterns). To choose the best potential move to be evaluated by the tabu-search based algorithm two more metrics $C_{S S D}$ and $M_{S S D}$ are introduced as follows.

The current spatial sharing degree $C_{S S D}\left(R_{i}, F R_{j}, I P\right)$ of a register $R_{i}$ for a module-type $F R_{j}$ and input port $I P$ is the number of modules of the respective module-type which are fed by register $R_{i}$ in the current testable solution. The maximum spatial sharing degree $M_{S S D}\left(R_{i}, F R_{j}, I P\right)$ of a register $R_{i}$ for a module-type $F R_{j}$ and input port $I P$ is the number of modules of the respective module-type which can be fed by the respective register using the data path interconnections. The metric which decides on the move for the respective register is $\Delta_{i}\left(F R_{j}, I P\right)=M_{S S D}\left(R_{i}, F R_{j}, I P\right)$ - $C_{S S D}\left(R_{i}, F R_{j}, I P\right)$. Having computed $\Delta_{i}\left(F R_{j}, I P\right)$ for each module-type $F R_{j}$, the transfer of test pattern generationfunctions for input port $I P$ of module-type $F R_{j}$ to register $R_{i}$ is performed such that $\Delta_{i}\left(F R_{j}, I P\right)$ is maximal. If there are two ore more input ports or module-types for which $\Delta_{i}$ is maximal then the decision is taken based on the maximum value of $M_{S S D}\left(R_{i}, F R_{j}, I P\right)$.

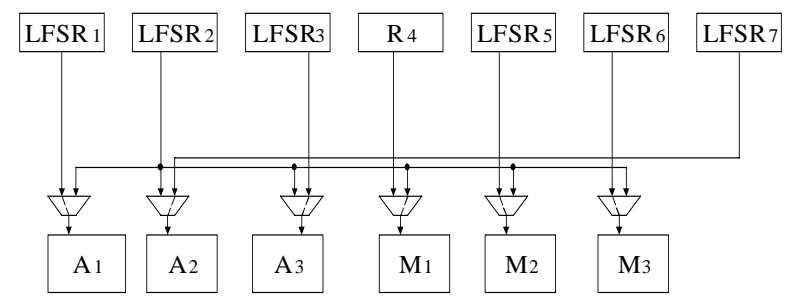

Figure 2. Partially specified testable design

In Figure 2 register $R_{2}$ is configured as LFSR and generates test vectors for the left input port of $M_{l}$. It can also generate test patterns for the left input port of $A_{1}$ and $A_{2}$, the right input port of $A_{3}$ and left input port of $M_{2}$ and $M_{3}$. We compute $\Delta_{2}\left(A_{t y p e}\right.$, Left $), \Delta_{2}\left(A_{\text {type }}\right.$, Right $)$, and $\Delta_{2}\left(M_{\text {type }}, L e f t\right)$. Since $R_{2}$ already performs pattern generation for the left input port of $M_{\text {type }}$ we obtain $\Delta_{2}\left(M_{\text {type }}, L e f t\right)=3-1=2$. In the same way $\Delta_{2}\left(A_{\text {type }}, L e f t\right)=2$ $0=2$ and $\Delta_{2}\left(A_{\text {type }}\right.$, Right $)=1-0=1$. Since $\Delta_{2}\left(M_{\text {type }}\right.$, Left $)=$ $\Delta_{2}\left(A_{\text {type }}\right.$, Left $)$ and $M_{S S D}\left(R_{2}, M_{\text {type }}\right.$, Left $)>M_{S S D}\left(R_{2}, A_{\text {type }}\right.$, Left $)$, the test pattern generation for $M_{\text {type }}$ is moved to $R_{2}$ and the area overhead is reduced because $R_{5}$ and $R_{6}$ do not perform test pattern generation as shown in Figure 3.

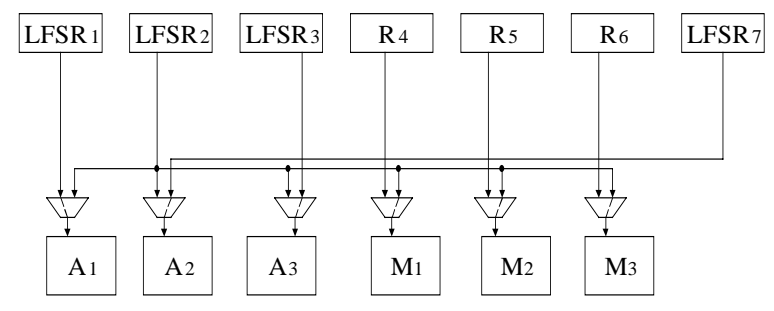

Figure 3. Partially specified testable design after the move of test pattern generation functions to $R_{2}$ 


\section{Incremental test scheduling algorithm}

In the previous section we have described how partially specified testable designs are generated from one solution to another reducing test area overhead of test pattern generators. At this stage test compatibility classes are not complete. Many modules are not sharing test resources and they can be introduced in the already existing test compatibility classes. The test compatibility classes are completed such that the number of potential signature analysis registers is increased as described in subsection 4.1. Modules with different physical information will require different test times to satisfy required fault coverage. Thus, the incremental test scheduling problem will deal with unequal test lengths as described in subsection 4.2.

\subsection{Generation of global test incompatibility graph}

In a partially specified testable solution all the modules are provided with their test pattern generators. Using the incompatibility between different modules in the partially specified testable design, an algorithm that guarantees the minimum number of test compatibility classes incompatible one with each other and an increase in the ORS of each test compatibility class is described. The creation of the global test incompatibility graph $(G-T I G)$ where nodes are TCCs is done in three phases as follows.

In the first phase the test compatibility classes for each module-type are formed. If two modules of the same type share a test resource then an edge between the respective two modules is added in the local test incompatibility graph $(L-T I G)$ of the respective module-type. The number of $L$-TIGs is $n_{\text {res. }}$. The L-TIGs are partitioned using an efficient graph partitioning algorithm [7, algorithm 1]. In the second phase the sharing of test resources between modules of different module-types is examined. If $M_{a}$ of module-type $m l$ is incompatible with $M_{b}$ of module-type $m 2$ and there is an edge in $G-T I G$ between a $T C C_{x, m l}$ and $T C C_{y, m 2}$ then $M_{a}$ is added to class $x$ and $M_{b}$ is added to class $y$. Otherwise $M_{a}$ is added class $i$ such that:

$f\left(T C C_{i, m l}, M_{a}\right)=\left|\operatorname{ORS}\left(T C C_{i, m l}+M_{a}\right)\right|-\left|\operatorname{ORS}\left(T C C_{i, m l}\right)\right|$

is maximal and a new edge between $T C C_{i, m l}$ and $T C C_{y, m 2}$, where $y$ is the class where $M_{b}$ was assigned, is added to $G$-TIG. Using this new measure $(f)$ an increase in the number of potential signature registers is assured. This increase will be used by the incremental test scheduling algorithm as outlined in the next subsection. In the third phase modules $M_{a}$ of module-type $m l$ that are present in the data path but do not share any test registers are added to a test class $i$ such that $f\left(T C C_{i, m l}, M_{a}\right)$ is maximal.

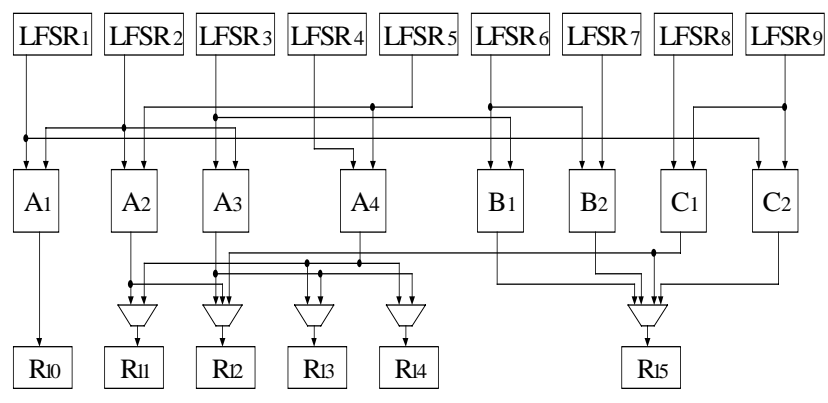

Figure 4. Data path example demonstrating the generation of G-TIG

Generation of G-TIG algorithm is illustrated by considering the data path in Figure 4. In the first step the $L-T I G s$ are created. Since modules that belong to $B_{t y p e}$ and $C_{\text {type }}$ do not share test registers with same-type modules, $L$-TIGs for these two module-types are void. Modules $A_{I}$ and $A_{2}$ cannot be tested at the same time because $L F S R_{2}$ is a shared test resource. Thus an incompatibility edge between $A_{1}$ and $A_{2}$ is introduced in the $L-T I G$ of $A_{\text {type }}$. Another edge added to the same $L-T I G$ is between $A_{2}$ and $A_{3}$ due to the sharing of test register $L F S R_{2}$. So far, the test classes for $A_{\text {type }}$ are $T C C_{0,0}=\left\{A_{1}, A_{3}\right\}$ and $T C C_{1,0}=\left\{A_{2}\right\}(0$ is the index of $A_{\text {type }}$ ). In the second step $T C C_{0,1}$ (1 is the index of $\left.B_{t y p e}\right)$ and $T C C_{0,2}\left(2\right.$ is the index of $\left.C_{t y p e}\right)$ are created. $B_{1}$ and $B_{2}$ are assigned to $T C C_{0,1}$ whilst $C_{1}$ and $C_{2}$ are assigned to $T C C_{0,2}$. Due to the sharing of $L_{F S R_{3}}$ between $A_{3}$ and $B_{1}$ an edge between $T C C_{0,0}$ and $T C C_{0,1}$ is added. Similarly an edge between $T C C_{0,0}$ and $T C C_{0,2}$ is added due to sharing of $L F S R_{l}$ between $A_{1}$ and $C_{2}$. In the third step, $A_{4}$ is assigned to one of the TCCs of $A_{\text {type }}$. The computed measures are $f\left(T C C_{0,0}, A_{4}\right)=1$ and $f\left(T C C_{1,0}, A_{4}\right)=$ 2. We choose $T C C_{1,0}$ due to higher increase in the size of ORS. The test classes are $T C C_{0,0}=\left\{A_{1}, A_{3}\right\}, T C C_{1,0}=\left\{A_{2}\right.$, $\left.A_{4}\right\}, T C C_{0,1}=\left\{B_{1}, B_{2}\right\}$ and $T C C_{0,2}=\left\{C_{1}, C_{2}\right\}$.

\subsection{Test scheduling and signature analysis register allocation}

The test scheduling algorithm for partitioned testing with run to completion reported in [7, algorithm $2 \mathrm{M}]$ is modified in order to schedule tests and allocate signature analysis registers simultaneously. If two test compatibility classes use the same signature register at different test times area overhead is reduced and the test control is simplified because the signature does not have to be shifted out. The concurrent test set (CTS) [7] contains the currently active tests. A similar data structure called busy register set $(B R S)$ keeps the test registers that are currently performing signature analysis. When the shortest currently 
active tests are completed and CTS is updated, the registers that performed signature analysis for the respective tests are removed from $B R S$. If $T C C_{i, j}$ is compatible with all tests from $C T S$, but all the registers from $\operatorname{ORS}\left(T C C_{i, j}\right)$ are present in $B R S$, then the test $T C C_{i, j}$ is postponed until at least one register that can perform signature analysis for $T C C_{i, j}$ is removed from $B R S$. One more data structure, used register set $(U R S)$, stores the test registers that have already performed signature analysis at a previous test time. Choosing a register from URS to perform signature analysis for the currently scheduled test leads to a reduction in area overhead. Another reason for reusing the previous signature analysis registers is that the signature does not have to be shifted out, unless the test registers have to be configured at a later stage as test pattern generators. The increase in fault-escape probability caused by not shifting out the signatures after the completion of each test is compensated by the decrease in fault escape probability achieved by checking the output responses of modules from the same test compatibility class using the $n$-input $k$-bit comparators.

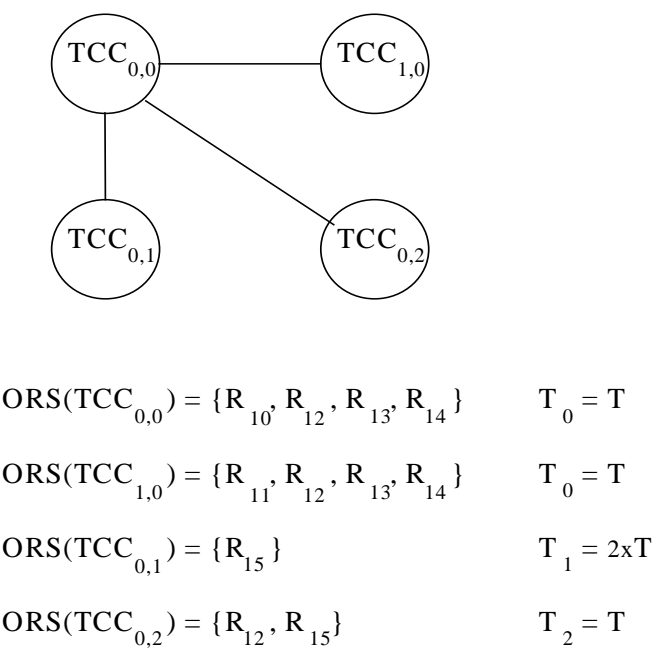

Figure 5. G-TIG for the data path example of Figure 4

To illustrate the above the $G-T I G$ of the data path from Figure 4 is shown in Figure 5. It is assumed that the number of test patterns to test $A_{\text {type }}$ and $C_{\text {type }}$ modules is equal $T_{0}=T_{2}=T$, whilst the number of test patterns to test $B_{\text {type }}$ modules is double $T_{l}=2 \mathrm{x} T$, with $T$ a reasonable high integer. The first test to be scheduled is $T C C_{0,0}$ at test time 0 . The signature analysis register is chosen from $\operatorname{ORS}\left(T_{C C_{0,0}}\right)$ such that its fanin is maximum. We choose $R_{12}$ to perform the signature analysis for $T C C_{0,0}$. At test time $T$, the $C T S$ is modified to reflect the removal of the

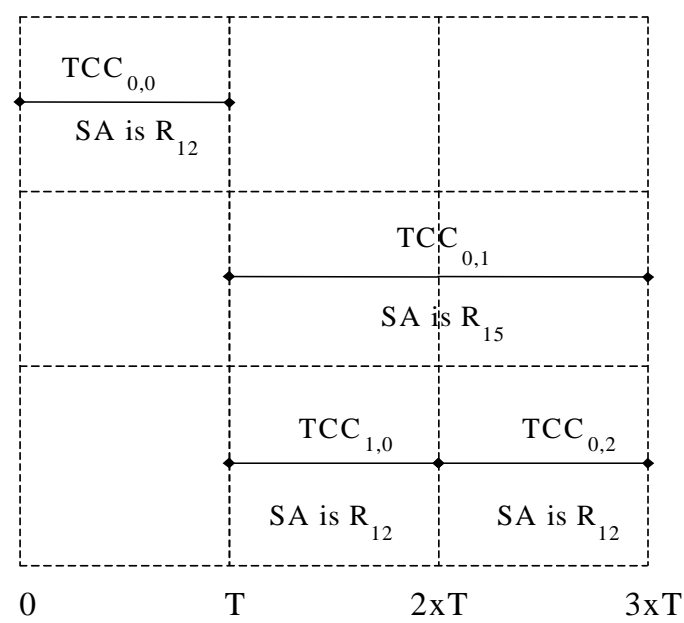

Figure 6. Test schedule for the data path
example of Figure 4

completed test $T C C_{0,0}$ and $R_{12}$ is removed from $B R S$ and added to $U R S$. At this point $T C C_{0,1}$ is scheduled. The register $R_{15}$ is assigned to perform signature analysis for $T C C_{0,1}$. At the same time $T C C_{1,0}$ is scheduled and register $R_{I 2}$ is considered for analyzing the responses of $T C C_{I, 0}$ because it is the only register from $\operatorname{ORS}\left(T C C_{1,0}\right)$ that has already been modified to a MISR (belongs to URS). An attempt to schedule $T C C_{0,2}$ fails because both registers that can perform signature analysis for this test class $\left(R_{15}\right.$ and $R_{12}$ ) belong to $B R S$. The shortest currently active test is completed at test time $2 \mathrm{x} T$. Register $R_{12}$ is removed from $B R S$ and another attempt to schedule $T C C_{0,2}$ will succeed because $R_{12}$ will be available to perform signature analysis. At test time $3 \times T$ all the currently active tests are completed. Note that only two test registers are required to perform signature analysis. A graphical representation of the test schedule is presented in Figure 6.

\section{Testable design space exploration phases}

In section 3 the local neighbourhood search of the testable design space was outlined. The test application time of the fully testable design computed using the incremental test scheduling algorithm given in section 4 is used to guide the testable design space exploration. Due to the topology of the testable design space and the local neighbourhood definition, in order to reach the best testable solution in low computational time the optimization is carried out in two phases. The need of this two-phase optimization process is justified by the three dimensional testable design space. There might be several testable designs with the same test application time but different BIST area overheads. Clearly in practice only the 
minimum test application time design with the smallest BIST area overhead is sought. Thus the primary goal is to minimize the test application time and the second order goal is to reduce the BIST area overhead. The BIST area overhead is computed in terms of the number of gates required to modify a register in a BIST register and the number of gates implementing $n$-input $k$-bit comparators. The testable design space is shown in Figure 7. Various testable designs (D1 to D7) with identical test application have different BIST area overheads. The best test application time testable design with minimum BIST area overhead is sought in this three dimensional testable design space.

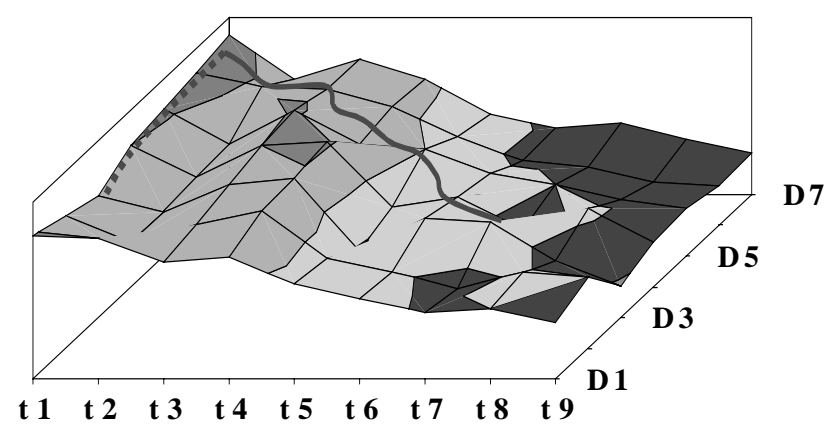

Figure 7. Testable design space exploration

The two optimization phases are:

(i)

Test application time reduction phase

(ii) BIST area overhead reduction phase

In the first optimization phase the minimum test application time designs are sought. If two solutions have same test application time then the solution with higher area overhead is chosen. This is due to the topology of the testable design space where higher BIST area overhead testable designs can lead in fewer moves to a testable design with lower test application time. If any of the neighbour solutions has lower test application then it is accepted regardless of the BIST area overhead. In Figure 7 the test application time reduction phase is illustrated by the curve with a continuos line. Having reached the minimum test application time design in the first optimization phase, during the second optimization phase testable designs with lower BIST area overhead are sought. If two testable designs have the same test application then the testable design with lower BIST area overhead is chosen. If any of the testable designs has higher test application it is rejected regardless of the BIST area overhead. The curve with the dashed line in Figure 7 shows the BIST area overhead reduction phase. Using this two-phase optimization strategy the computational time required for testable design space exploration time is reduced as shown in the next section.

\section{Experimental results}

The proposed BIST hardware insertion algorithm based on TCCs has been implemented on SUN SPARC 20 workstation using 6000 lines of $\mathrm{C}++$ code. Table 1 shows a comparison of experimental results in terms of test application time (TAT) and BIST area overhead obtained for the BIST embedding methodology and the proposed BIST methodology based on TCCs. The results for the BIST embedding methodology are obtained by considering $n_{\text {mod }}=n_{\text {res }}$. The RTL data paths are generated using a high-level synthesis system [14]. The first column shows the design name and the execution time constraint which varies from 10 to 16 in the case of 8 point DCT and 30 to 38 in the case of 32 point DCT. The next two columns show the number of modules (MOD) in terms of multipliers $\left(^{*}\right)$ and adders $(+)$ and the number of registers (REG) obtained from high level synthesis. The TAT for adders and multipliers was assumed $\mathrm{T}_{+}=T$ and $\mathrm{T}_{*}=4 \mathrm{x} T$ with $T$ a reasonable high integer. The first six rows show the experimental results for the 8 point DCT. In the first four rows the BIST embedding methodology produces testable data paths where TAT is not minimal. Applying the proposed BIST methodology based on TCCs, TAT is reduced by $20 \%$ from $5 \times T$ to $4 \times T$. The reduction in TAT produces higher area overheads in some cases like 8DCT_10. However, the primary goal of reducing the TAT is achieved. Furthermore the number of BIST registers embedded in data path is reduced which leads to faster solutions. In the case of 8DCT_11, both TAT and BIST area overhead are reduced. The number of LFSRs (represented by L in Table 1) is reduced from 8 to 6 whilst the number of MISRS (represented by $\mathrm{M}$ in Table 1) is reduced from 7 to 2 . In the proposed BIST methodology a single 3 input 8 bit comparator $(C 3)$ and a single 4 input 8 bit comparator $(C 4)$ are introduced. However these two comparators do not have high area overhead, reduce the fault escape probability and are easy testable. For the last two 8 point DCT designs the proposed BIST methodology reduces the BIST area overhead by $27 \%$ and $12 \%$ respectively which clearly outlines the efficiency of the second order BIST area optimization goal. The last 6 rows of Table 1 show that the proposed methodology is capable of dealing with complex DSP circuits such as 32 point DCT. Due to a very high number of registers the minimal TAT is achieved by both methodologies. The reductions in BIST area overhead are significant varying from $35 \%$ up to $48 \%$. The algorithm has low computational time as shown in the last column of Table 1. For 8 point DCT the computational time varies from $1 \mathrm{~s}$ to $3 \mathrm{~s}$. For 32 point DCT designs the computational time is still very low. The excellent computational time is achieved due to use of intelligent local neighbourhood search of the testable design space and the two-phase optimization strategy. 


\begin{tabular}{|c|c|c|c|c|c|c|c|c|c|c|}
\hline \multirow[t]{3}{*}{ Design } & \multirow{3}{*}{$\begin{array}{l}\mathrm{M} \\
\mathrm{O} \\
\mathrm{D}\end{array}$} & \multirow{3}{*}{$\begin{array}{l}\text { R } \\
\text { E } \\
\text { G }\end{array}$} & \multicolumn{2}{|c|}{ BIST embedding } & \multicolumn{3}{|c|}{ BIST methodology based on TCC } & \multirow{3}{*}{$\begin{array}{c}\text { TAT } \\
\text { red. } \%\end{array}$} & \multirow{3}{*}{$\begin{array}{l}\text { BIST } \\
\text { Area } \\
\text { red. \% }\end{array}$} & \multirow{3}{*}{$\begin{array}{c}\text { CPU } \\
\text { Time } \\
\text { (s) }\end{array}$} \\
\hline & & & \multirow[t]{2}{*}{ TAT } & \multirow{2}{*}{$\begin{array}{c}\text { Test } \\
\text { Resources }\end{array}$} & \multirow[t]{2}{*}{ TAT } & \multicolumn{2}{|c|}{ Test resources } & & & \\
\hline & & & & & & BIST REG & 8 bit comparators & & & \\
\hline 8DCT_10 & $4^{*}, 4+$ & 15 & $5 \times T$ & $7 \mathrm{~L}, 6 \mathrm{M}$ & $4 \times T$ & $8 \mathrm{~L}, 3 \mathrm{M}$ & $2 \mathrm{C} 2,1 \mathrm{C} 4$ & 20 & -26.58 & 2.56 \\
\hline 8DCT_11 & $4 *, 3+$ & 15 & $5 \times T$ & $8 \mathrm{~L}, 7 \mathrm{M}$ & $4 \times T$ & $6 \mathrm{~L}, 2 \mathrm{M}$ & $1 \mathrm{C} 3,1 \mathrm{C} 4$ & 20 & 18.55 & 2.57 \\
\hline 8DCT_12 & $4 *, 3+$ & 16 & $5 \times T$ & $8 \mathrm{~L}, 7 \mathrm{M}$ & $4 \times T$ & $8 \mathrm{~L}, 2 \mathrm{M}$ & $1 \mathrm{C} 3,1 \mathrm{C} 4$ & 20 & -1.55 & 1.86 \\
\hline 8DCT_13 & $4 *, 4+$ & 16 & $5 \times T$ & $9 \mathrm{~L}, 5 \mathrm{M}$ & $4 \times T$ & $8 \mathrm{~L}, 2 \mathrm{M}$ & $2 \mathrm{C} 4$ & 20 & -4.03 & 2.67 \\
\hline 8DCT_14 & $3 *, 3+$ & 16 & $4 \times T$ & $9 \mathrm{~L}, 6 \mathrm{M}$ & $4 \times T$ & $5 \mathrm{~L}, 2 \mathrm{M}$ & $2 \mathrm{C} 3$ & 0 & 27.53 & 1.20 \\
\hline 8DCT_16 & $3^{*}, 2+$ & 16 & $4 \times T$ & $7 \mathrm{~L}, 5 \mathrm{M}$ & $4 \times T$ & $5 \mathrm{~L}, 2 \mathrm{M}$ & $1 \mathrm{C} 2,1 \mathrm{C} 3$ & 0 & 12.31 & 1.15 \\
\hline 32DCT_30 & $9 *, 12+$ & 60 & $4 \times T$ & $32 \mathrm{~L}, 21 \mathrm{M}$ & $4 \times T$ & $18 \mathrm{~L}, 2 \mathrm{M}$ & $1 \mathrm{C} 5,1 \mathrm{C} 7,1 \mathrm{C} 9$ & 0 & 44.48 & 129.7 \\
\hline 32DCT_31 & $9 *, 12+$ & 62 & $4 \times T$ & $33 \mathrm{~L}, 21 \mathrm{M}$ & $4 \times T$ & $19 \mathrm{~L}, 2 \mathrm{M}$ & $1 \mathrm{C} 5,1 \mathrm{C} 7,1 \mathrm{C} 9$ & 0 & 42.63 & 124.4 \\
\hline 32DCT_32 & $8^{*}, 12+$ & 62 & $4 \times T$ & $32 \mathrm{~L}, 20 \mathrm{M}$ & $4 \times T$ & $16 \mathrm{~L}, 2 \mathrm{M}$ & $1 \mathrm{C} 4,2 \mathrm{C} 8$ & 0 & 46.86 & 103.7 \\
\hline 32DCT_33 & $8 *, 11+$ & 62 & $4 \times T$ & $30 \mathrm{~L}, 19 \mathrm{M}$ & $4 \times T$ & $14 \mathrm{~L}, 2 \mathrm{M}$ & $1 \mathrm{C} 5,1 \mathrm{C} 6,1 \mathrm{C} 8$ & 0 & 48.40 & 55.00 \\
\hline 32DCT_37 & $8 *, 9+$ & 63 & $4 \times T$ & $26 \mathrm{~L}, 17 \mathrm{M}$ & $4 \times T$ & $16 \mathrm{~L}, 3 \mathrm{M}$ & $1 \mathrm{C} 3,1 \mathrm{C} 6,1 \mathrm{C} 8$ & 0 & 35.84 & 86.64 \\
\hline 32DCT_38 & $9 *, 9+$ & 59 & $4 \times T$ & $27 \mathrm{~L}, 18 \mathrm{M}$ & $4 \times T$ & $16 \mathrm{~L}, 2 \mathrm{M}$ & $1 \mathrm{C} 8,1 \mathrm{C} 9$ & 0 & 42.12 & 38.47 \\
\hline
\end{tabular}

Table 1. Experimental results for the BIST embedding and BIST methodology based on TCCs

\section{Conclusions}

New BIST methodology and BIST hardware insertion algorithms for synthesized data paths were presented. The methodology is based on grouping modules with same physical information in test compatibility classes. Input ports of compatible modules share test pattern generators in a partial intrusion BIST environment leading to savings in test application time. Module output responses from each test compatibility class are checked by an $n$-input $k$ bit comparator leading to lower area overhead. Nearoptimal testable design is obtained in low computational time by a two-phase tabu search-based testable design space exploration.

\section{References}

[1] V.D., Agrawal, C.R., Kime, and K.K. Saluja. A tutorial on built-n self - part 1: Principles. IEEE Design \& Test of Computers, pp. 73-92, March 1993.

[2] V.D., Agrawal, C.R., Kime, and K.K. Saluja. A tutorial on built-in self test - part 2: Applications. IEEE Design \& Test of Computers, pp. 69-77, June 1993.

[3] P.R. Chalsani, S. Bhawmik, A. Acharya, and P. Palchaudhuri. Design of testable VLSI circuits with minimum area overhead. In IEEE Transactions on Computers, 38(9), pp. 1460-1462, 1989.

[4] A. Basu, T.C. Wilson, D.K. Banerji, and J.C. Majithia. An approach to minimize testability for BILBO based built-in selftest. In Proc. 5th Int. Conf on VLSI Design, pp. 354-355, 1992.
[5] S.P. Lin, C.A. Njinda, and M.A. Breuer. Generating a family of testable designs using the BILBO methodology. In Journal of Electronic Testing: Theory and Applications (JETTA) 4, pp. 7189, April 1993.

[6] C.I.H. Chen. Graph partitioning for concurrent test scheduling in VLSI circuits. In Proc. $28^{\text {th }}$ Design Automation Conference, pp. 287-290, 1991.

[7] G.L. Craig, C.R. Kime, and K.K. Saluja. Test scheduling and control for VLSI built-in self-test. IEEE Transactions on Computers, 37(9), pp.1099-1109, 1988.

[8] W.B. Jone, C.A. Papachristou, and M. Pereira. A scheme for overlaying concurrent testing of VLSI circuits. In Proc. $26^{\text {th }}$ Design Automation Conference, pp. 531-536, June 1989.

[9] A. Orailoglu., and I.G. Harris. Test path generation and test scheduling for self-testable designs. In Proc. International Conference on Computer Design, pp. 528- 531, 1993.

[10] I.G. Harris, and A. Orailoglu. Fine-grained test concurrency in test scheduling for partial-intrusion BIST. In Proc. European Design \& Test Conference, pp. 119-123, 1994.

[11] S. Chiu, and C.A. Papachristou. A design for testability scheme with application to data path synthesis. In Proc. $28^{\text {th }}$ Design Automation Conference, pp. 271-277, June 1991.

[12] A.P. Stroele, and H.J. Wunderlich. Hardware-optimal test register insertion. IEEE Transactions on Computer-Aided Design of Integrated Circuits and Systems, 17(6), pp. 531-539, 1998.

[13] C.R. Reeves. Modern heuristic techniques for combinatorial problems, McGraw-Hill Book Company, 1995.

[14]P. Kollig, and B.M. Al-Hashimi. Simultaneous scheduling, allocation and binding in high level synthesis. IEE Electronics Letters, 33(18), pp. 1516-1518, 1997 